\title{
Innovation Research on Learning from Lv Qingsen Activities by Grass-root Party Organizations
}

\author{
Zhang Xilin ${ }^{1, a}$, Xu Shouchen ${ }^{2, b}$ \\ 1. State Grid Jilin Electric Power Company Limited Changchun Power Supply Company , \\ Changchun, 130021, China \\ 2. State Grid the Senior Training Center, Beijing, 100085, China \\ aemail: xilinzhang@21cn.com, bemail: shouchen-xu@sohu.com
}

\begin{abstract}
Keywords: Lv Qingsen; Electric Transmission Line; Traditional Chinese Values; Core Value of State Grid Corporation of China
\end{abstract}

\begin{abstract}
Innovation research is made on learning from Lv Qingsen activities by grass-root party organizations from Lv Qingsen spiritual connotation and its incentive effect on staff in electrical power system, planning on activities of sustaining and deepening learning practice on Lv Qingsen spirit, enlightenment from Lv Qingsen and being the staff in electrical power system as Lv Qingsen so as to improve the development of activities of sustaining and deepening learning practice on Lv Qingsen spirit by grass-root party organizations.
\end{abstract}

\section{Introduction}

Decision on Learning from Comrade Lv Qingsen in National Electrical Power System was made by State Electricity Regulatory Commission on June $30^{\text {th }}, 2010$ to call on the staff in national electrical power system to learn from Comrade Lv Qingsen. What is the core of Lv Qingsen spirit? What is the motivation for Comrade Lv Qingsen to create the extraordinary performance on the ordinary post? What shall we learn from Comrade Lv Qingsen and how to learn and what kind of effect should be achieved? How to implement Lv Qingsen spirit in the department, unit and also the post? How to be a good employee as Lv Qingsen? They are the questions that need to be answered urgently by the administrators in the grassroots units of State Grid Corporation of China so as to know how to learn from Comrade Lv Qingsen by themselves and how to lead the staff in the department to launch the activities on learning from Comrade Lv Qingsen effectively and steadily.

\section{Spiritual Connotation of Lv Qingsen and Its Incentive Effect on Staff in Electrical Power System ${ }^{[1]}$}

A. Spiritual connotation of Lv Qingsen

The spiritual connotation of Lv Qingsen is the perfect combination of Chinese traditional virtues and the core value of State Grid Corporation of China.

(a) Lv Qingsen spirit is the inheritance of Chinese traditional virtues

Comrade Lv Qingsen has guarded the electric transmission line from Hongshi to Baishan town for 31 years and he should trek more than $200 \mathrm{~km}$ for line patrolling in the high mountains and lofty hills through hardships by himself every month during these years so that the safe operation of electric transmission line from Hongshi to Baishan town is guaranteed for 31 years, which manifests the noble sentiments of staff in State Grid Corporation of China including devotion to duty, arduous struggle, assiduous study and quiet dedication.

(b) Lv Qingsen spirit is the concentrated reflection of the core value of State Grid Corporation of China

The words and deeds of Comrade Lv Qingsen can fully reflect the core value of State Grid Corporation of China including "integrity, responsibility, innovation and devotion". He should patrol the electric transmission line from Hongshi to Baishan town by himself, which really depends on his conscience and thus the 31 years' safe operation of electric transmission line from Hongshi to 
Baishan town is guaranteed due to his life creed of honesty and trustworthiness and highly-developed sense of responsibility.

(c) Motivation for Comrade Lv Qingsen to create the extraordinary performance on the ordinary post

The motivations for Comrade Lv Qingsen to guard the electric transmission line from Hongshi to Baishan town for 31 years and guarantee its safe operation are the goodness and persistence of Comrade Lv Qingsen, deep love for the electric transmission line from Hongshi to Baishan town, suffering in the mountains, external environment of family and enterprise, edification by traditional Chinese culture and cultivation of Party organization.

B. Incentive Effect of Lv Qingsen Spirit on Staff in Electrical Power System

Learning from Comrade Lv Qingsen is to learn his spiritual connotation and carry forward Lv Qingsen spirit through working. Firstly, learn his responsibility consciousness for being loyal to the Party, motherland and people. Take the requirements of enterprise and users as our own missions, perform our duties and practice the service tenets of "People's electric utility for people" . Secondly, learn his passionate in his job and courage to forge ahead. Establish your self in your post and love what you do and master what you specialize in and realize your life value through strong skills and top-ranking performance. Thirdly, learn his noble sentiments of arduous struggle and selfless contribution. Feel grateful for the enterprise and transform the gratitude into work motivation and reward the enterprise with "master" feeling. Fourthly, learn his enquirying minds of overcoming difficulties and carefulness. Have the courage to face difficulties and challenges and assault fortified positions, forge ahead despite difficulties, face difficulties, explore energetically and study diligently during the important period for development of power grid and enterprise innovation.

\section{Plans for Activities of Sustaining and Deepening Learning Practice on Lv Qingsen Spirit ${ }^{[2]}$}

\section{A. About the model of Lv Qingsen}

(a) Distinctive characteristics of the times of the model

It is believed in the social learning theory of Bandura, an educational psychologist that "people can not only learn from direct experience, but also from observing the behaviors of other people, namely, the model and then acquire the indirect experience. Learning from observation plays an important role in human learning and generally speaking, the behavior of the awarded model is more likely to be imitated by the observer. "The theory of educational psychology provides the theoretical foundation for cultivating the model of Lv Qingsen.

(b) Maintaining the relative stability of the model

It is also believed in the social learning theory of Bandura, an educational psychologist that "most of the human activities are learned from observation which is dominated by a series of interdependent psychological processes (attention, maintenance, action repetition and motivation)." It is posed in the theory of educational psychology that social learning needs relative stability, the essential factors for the social learning including attention, maintenance and action repetition of the learner can only be generated through the continuous and stable publicity of Lv Qingsen spirit; the model should be stable and similarly the spiritual connotation of the model should also be stable.

(c) Maintaining the modesty of Lv Qingsen, the electric transmission line worker in life and work

Reverse psychology is the biggest bottleneck in the implementation of ideological and political work and also it is the psychological problem that should be faced in sustaining and deepening learning practice on Lv Qingsen spirit. The contemporary characteristics of Lv Qingsen spirit have been summarized and abstracted and the perfect human nature and the lofty professional ethic of Lv Qingsen are expressed fully; maintaining the modesty of Lv Qingsen, the electric transmission line worker in life and work in sustaining and deepening learning practice on Lv Qingsen spirit is a forceful measure to improve the recognition degree of learner so as to overcome reverse psychology and guarantee activity effect; only when the model is highly approved by the learner, the external conditions of the model can become the motivation for internal conversion of learner's thinking and 
his study.

B. Spiritual Connotation of Lv Qingsen as Major Components of Enterprise Culture of State Grid Corporation of China

Value is one of the major components of enterprise culture. The spiritual connotations of LV Qingsen were summarized and extracted in Decision on Learning from Comrade Lv Qingsen in National Electrical Power System by State Electricity Regulatory Commission. Firstly, learn his responsibility consciousness for being loyal to the Party, motherland and people. Take the requirements of enterprise and users as his mission, perform his duties and practice the service tenets of "People's electric utility for people". Secondly, learn his passionate in his job and courage to forge ahead. Establish himself in his post and love what he do and master what he specialize in and realize his life value through strong skills and top-ranking performance. Thirdly, learn his noble sentiments of arduous struggle and selfless contribution. Feel grateful for the enterprise and transform the gratitude into work motivation and reward the enterprise with "master" feeling. Fourthly, learn his spirit of inquiry in overcoming difficulties and carefulness.

C. Cultivation of the Examples around

The sustaining and deepening learning practice on Lv Qingsen spirit can be promoted through combining the model and the examples fostered around. The examples around have similar working environment, working content, working target and assessment criterion with the learners and thus they can catch the attention of the learners and make them keep their attention. Therefore, examples around are more easily to be the standard for staff behavior and target for comparing, learning and surpassing and thus enthusiasm and creativity are combined; the potentials for all the staff to complete their jobs perfectly are motivated so that internal cause and external cause are combined; the examples around have real effects and thus the harmonious development between psychology and cognition of learners is realized.

\section{Enlightenments Brought by Lv Qingsen ${ }^{[3]}$}

A. Every post can be respected by the enterprise and society through diligent and hard work

Everyone aspires to be respected and it is the ideal goal for highly developed society that everyone is equal and respected. In human society, there must be social division of labor that is both the need of human survival and symbol of society progress. However, with the continuous development of market economy, the economic income and social status gaps are widened constantly due to the different social division of labor, meanwhile, we start to go far away from the ideal goal of highly developed society that everyone is equal and should be respected gradually and people's value changes greatly.

B. Treating Success and Failure with Usual Mind Is the Footstone of Happiness

Lv Qingsen works in very tough environment with few inhabitants and many wild animals and the mountains are high and forests are thick and also extreme weathers often happen. The maintenance work for electric transmission line is very monotonous and dull, however, Lv Qingsen treats the working environment and post with usual mind and chooses to be brave, responsible and insist. Although Lv Qingsen's job is very hard, we can still feel that his rich inner world and happy subjective feeling through his deep love for work and life, clangorous song in the deep mountains and forests and his excellent performance.

C. Happiness is Subjective Feeling

The post of Lv Qingsen is very arduous and even sometimes the working environment is very dangerous, however, Lv Qingsen is very happy in his inner world, which comes from his goodness and straightforward character, his gratitude for the enterprise, his sense of pride for completing job, his sense of achievement for devoting to the society and affection from family and collectivity.

D. Extraordinary Performance Can be Created on Ordinary Post

The maintenance work for $66 \mathrm{kV}$ electric transmission line from Hongshi to Baishan town is very ordinary, however, the $66 \mathrm{kV}$ electric transmission line from Hongshi to Baishan town is operated with no defects and abnormalities for more than 30 years through the hard work of Lv Qingsen, 
which is an extraordinary performance. Lv Qingsen insists to work under tough environment for more than 30 years, which shows his greatness in ordinary.

\section{Conclusion}

If the grassroots units in the State Grid Corporation of China want to launch the activities in learning from Comrade Lv Qingsen, the Chinese traditional virtues and core value of State Grid Corporation of China should be combined perfectly, which is the spiritual connotation of $\mathrm{LV}$ Qingsen. Create activity form and carrier that are suitable for the personnel structure and production characteristic of the department within the framework of the guidance of State Electricity Regulatory Commission so as to guarantee the effectiveness of the learning activity and the durability of the role model power.

\section{References}

[1] Li Yiling, Zhang Xilin. Discussion on the Spiritual Connotation of Lv Qingsen and Its Incentive Effect on Staff in Electrical Power System [J]. China Electric Power Education,2010,28:231-232.

[2] Zhang Xilin, Wang Shuo, Li Linze. Plan for Activity of Sustaining and Deepening Learning Practice on Lv Qingsen Spirit [J]. China Electric Power Education, 2011, 27:23-24.

[3] Zhang Xilin. Enlightenment Brought by Lv Qingsen [J]. Jilin Electric Power,2011,12:12-13.

[4] Zhang Xilin, Wang Shuo, Li Linze. Being the Staff in Electrical Power System as Lv Qingsen [J]. Power human resource,2012,3:80-82. 